\title{
ON THE WEDDERBURN NORM CONDITION FOR CYCLIC ALGEBRAS*
}

\author{
BY A. A. ALBERT
}

1. Introduction. Let $F$ be any non-modular field, $i$ a root of a cyclic equation in $F$ of degree $n$ and with roots $\theta^{r}(i)$. Suppose that $A$ is a cyclic algebra with basis

where

$$
i^{r} y^{s}, \quad(r, s=0,1, \cdots, n-1),
$$

$$
y^{r} i=\theta^{r}(i) y^{r}, y^{n}=\gamma \text { in } F .
$$

J. H. M. Wedderburn has proved $\dagger$ that $A$ is a division algebra if $\gamma^{r}$ is not the norm, $N(a)$, of any $a$ in $F(i)$ for every positive integer $r$ less than $n$. It has never been shown, however, that this condition is a necessary one; but the problem of finding complete necessary and sufficient conditions has been reduced to the case $n$ a power of a single prime. $\ddagger$

In the present paper cyclic algebras of order sixteen with the corresponding cyclic quartic in its canonical form $\S$

$$
\phi(\omega) \equiv \dot{\omega}^{4}+2 \nu\left(1+\Delta^{2}\right) \omega^{2}+\nu^{2} \Delta^{2}\left(1+\Delta^{2}\right)=0
$$

such that $\nu$ and $\Delta$ are in $F$, and $\tau=1+\Delta^{2}$ is not the square of any quantity of $F$, are considered. The norm $N(a)$ of a polynomial in $i$ is a rather complicated quartic form in four variables, yet we can secure the result that $\gamma^{2}=N(a)$ if and only if $\gamma=\alpha^{2}-\beta^{2} \tau$ for $\alpha$ and $\beta$ in $F$, a curious property of cyclic quartic fields. When the above equation is satisfied the algebra $A$ is expressible as a direct product of two generalized quaternion algebras. Necessary and sufficient conditions are secured that our algebras $A$ of order sixteen be division algebras, and it is shown that for the particularly interesting case where $F$ is the field of all rational numbers the Wedderburn condition is necessary as well as sufficient.

* Presented to the Society, December 30, 1930.

$\dagger$ Transactions of this Society, vol. 15 (1914), pp. 162-166.

$\ddagger$ See a paper by the author, On direct products, cyclic algebras, and pure Riemann matrices, to appear in the Transactions of this Society, January, 1931.

$\S$ See R. Garver, Quartic equations with certain groups, Annals of Mathematics, vol. 29 (1928), pp. 47-51. 
2. The Basic Theorem. Let $F(x)$ be a cyclic quartic field. Then it is known (loc. cit.) that $F(x)=F(i)$, where $i$ satisfies the equation

$$
\phi(\omega) \equiv \omega^{4}+2 \nu \tau \omega^{2}+\nu^{2} \Delta^{2} \tau=0,
$$

with $\tau=1+\Delta^{2}$ not the square of any quantity of $F$ and

$$
\nu \neq 0, \tau, \Delta \neq 0
$$

all in $F$. Moreover if we define $u$ by the equation

$$
i^{2}=\nu(u-\tau)
$$

then

$$
u^{2}=\tau, \quad \theta(i)=\frac{i}{\Delta}(u+1),
$$

is the polynomial whose iteratives $i=\theta^{0}(i)=\theta^{4}(i), \theta(i), \theta^{2}(i)=-i$, $\theta^{3}(i)=\theta(-i)=-\theta(i)$ give the four roots in $F(i)$ of $\phi(\omega)=0$. Every quantity of $F(i)$ is expressible in the form

$$
a=a_{1}+a_{2} i, \quad\left(a_{1} \text { and } a_{2} \text { in } F(u)\right),
$$

and $a=0$ if and only if $a_{1}=a_{2}=0$. A quantity .

$$
a_{1}=\alpha_{1}+\alpha_{2} u, \quad\left(\alpha_{1} \text { and } \alpha_{2} \text { in } F\right),
$$

is zero if and only if $\alpha_{1}=\alpha_{2}=0$; and similarly

$$
\alpha_{1}^{2}-\alpha_{2}^{2} \tau
$$

vanishes if and only if $\alpha_{1}=\alpha_{2}=0$ by our restriction on $\tau$.

We shall use repeatedly the following simple lemma.

LEMMA 1. Every product of a finite number of scalars of the forms

$$
\begin{aligned}
& \lambda^{2}-\mu^{2} \tau, \\
& \left(\lambda^{2}-\mu^{2} \tau\right)^{-1}, \quad \lambda^{2}-\mu^{2} \tau \neq 0,
\end{aligned}
$$

with $\lambda$ and $\mu$ in $F$, is expressible in the form (8) for $\lambda$ and $\mu$ in $F$.

The truth of this is evident since

$$
\left(\lambda_{1}+\mu_{1} u\right)\left(\lambda_{2}+\mu_{2} u\right)=\left(\lambda_{1} \mu_{1}+\lambda_{2} \mu_{2} \tau\right)+\left(\lambda_{1} \mu_{2}+\lambda_{2} \mu_{1}\right) u,
$$

and hence 
(9) $\left(\lambda_{1}^{2}-\mu_{1}^{2} \tau\right)\left(\lambda_{2}^{2}-\mu_{2}^{2} \tau\right)=\left(\lambda_{1} \mu_{1}+\lambda_{2} \mu_{2} \tau\right)^{2}-\left(\lambda_{1} \mu_{2}+\lambda_{2} \mu_{1}\right)^{2} \tau$;

while if $\epsilon=\lambda^{2}-\mu^{2} \tau \neq 0$, then

$$
\epsilon^{-1}=\epsilon^{-2} \epsilon=\epsilon^{-2}\left(\lambda^{2}-\mu^{2} \tau\right)=\left(\lambda \epsilon^{-1}\right)^{2}-\left(\mu \epsilon^{-1}\right)^{2} \tau .
$$

Let us now assume that $\gamma \neq 0$ is a scalar in $F$, such that $\gamma^{2}=N(a)$, where $a$ is in the cyclic field $F(i)$. We may write $a^{(r)}=a\left[\theta^{r}(i)\right], \quad(r=0,1, \cdots)$, whence $a^{\prime \prime}=a(-i)$. Then $u^{\prime}$ $=-u$; and if $a_{1}$ is in $F(u)$ so that $a_{1}$ has the form $a_{1}=\alpha_{1}+\alpha_{2} u$, we have $a_{1}=a_{1}^{\prime \prime}$ and

$$
N\left(a_{1}\right)=a_{1} a_{1}^{\prime} a_{1}^{\prime \prime} a_{1}^{\prime \prime \prime}=\left(a_{1} a_{1}^{\prime}\right)^{2}=\left(\alpha_{1}^{2}-\alpha_{2}^{2} \tau\right)^{2} .
$$

Let us write $\gamma^{2}=N(a)$, where

$$
a=a_{2}+a_{3} i, \quad\left(a_{2} \text { and } a_{3} \text { in } F(u)\right) .
$$

We shall first consider the case $a_{3}=0$. Then $a=a_{2}=\alpha_{3}+\alpha_{4} u$, and

$$
\gamma^{2}=\left(\alpha_{3}^{2}-\alpha_{4}^{2} \tau\right)^{2} .
$$

This equation in a field $F$ implies that

$$
\gamma= \pm\left(\alpha_{3}^{2}-\alpha_{4}^{2} \tau\right)
$$

If $\gamma=\alpha_{3}^{2}-\alpha_{4}^{2} \tau$, we have expressed $\gamma$ in the form

$$
\gamma=\alpha^{2}-\beta^{2} \tau
$$

with $\alpha$ and $\beta$ in $F$, the result desired. Since $\tau=1+\Delta^{2}$, we have

$$
-1=\Delta^{2}-\tau \text {. }
$$

Hence if $\gamma=-\left(\alpha_{3}^{2}-\alpha_{4}{ }^{2} \tau\right)$, then $\gamma=\left(\Delta^{2}-\tau\right)\left(\alpha_{3}^{2}-\alpha_{4}{ }^{2} \tau\right)$; and, by Lemma 1, $\gamma$ has again the desired form (15).

Next let $a_{3} \neq 0$. Then, if $a_{3}=\lambda_{3}+\lambda_{4} u, a_{1}=a_{3}^{-1} a_{2}$, we have

$$
N(a)=N\left[a_{3}\left(a_{1}+i\right)\right]=\left(\lambda_{3}^{2}-\lambda_{4}^{2} \tau\right)^{2} N\left(a_{1}+i\right) .
$$

Let $\delta=\gamma\left(\lambda_{3}^{2}-\lambda_{4}^{2} \tau\right)^{-1}$. Then

$$
\delta^{2}=\gamma^{2}\left(\lambda_{3}^{2}-\lambda_{4}^{2} \tau\right)^{-2}=N\left(a_{1}+i\right) .
$$

But if $b=a_{1}+i$, then $\delta^{2}=\left(b b^{\prime \prime}\right)\left(b b^{\prime \prime}\right)^{\prime}$ so that if $w=\delta\left[\left(b b^{\prime \prime}\right)^{\prime}\right]^{-1}$, then $\delta=\delta^{\prime}=w^{\prime} b b^{\prime \prime}$. It follows that $\delta^{2}=w w^{\prime} N(b)=w w^{\prime} \delta^{2}$. Hence

$$
w w^{\prime}=1, w=b b^{\prime \prime} \delta^{-1}, b b^{\prime \prime}=\delta w,
$$


where $w=b b^{\prime \prime} \delta^{-1}=\xi_{1}+\xi_{2} u$ is in $F(u)$. If $a_{1}=\alpha_{1}+\alpha_{2} u, \alpha_{1}$ and $\alpha_{2}$ in $F$, we have, by (3),

$$
\begin{aligned}
b b^{\prime \prime} & =a_{1}{ }^{2}-i^{2}=\alpha_{1}^{2}+\alpha_{2}^{2} \tau+2 \alpha_{1} \alpha_{2} u-\nu(u-\tau) \\
& =\left(\alpha_{1}^{2}+\alpha_{2}^{2} \tau+\nu \tau\right)+\left(2 \alpha_{1} \alpha_{2}-\nu\right) u .
\end{aligned}
$$

From the linear independence of 1 and $u$ this implies

$$
\alpha_{1}^{2}+\alpha_{2}^{2} \tau+\nu \tau=\delta \xi_{1}, 2 \alpha_{1} \alpha_{2}-\nu=\delta \xi_{2} .
$$

We obtain $2 \alpha_{1} \alpha_{2} \tau-\nu \tau=\delta \xi_{2} \tau$, and by addition

$$
\alpha_{1}^{2}+2 \alpha_{1} \alpha_{2} \tau+\alpha_{2}^{2} \tau=\delta\left(\xi_{1}+\xi_{2} \tau\right)
$$

Since $1-\tau=-\Delta^{2}$, if we complete the square in (21), it becomes

$$
\begin{aligned}
\left(\alpha_{1}+\alpha_{2} \tau\right)^{2}+\alpha_{2}^{2}\left(\tau-\tau^{2}\right) & =\left(\alpha_{1}+\alpha_{2} \tau\right)^{2}-\left(\alpha_{2} \Delta\right)^{2} \tau \\
& =\delta\left(\xi_{1}+\xi_{2} \tau\right)
\end{aligned}
$$

Consider now the equation $w w^{\prime}=1$, or

$$
\xi_{1}^{2}-\xi_{2}^{2} \tau=1, \xi_{2}^{2} \tau=\left(\xi_{1}+1\right)\left(\xi_{1}-1\right) .
$$

Let $\xi_{1}-1=2 \pi, \xi_{1}+1=2 \sigma$. Then

$$
4 \sigma \pi=\xi_{2}^{2} \tau
$$

Suppose first that $\xi_{1}+1=0$ so that $\sigma=0$ and $\xi_{2}=0$. Then $\xi_{1}+\xi_{2} \tau=\xi_{1}=-1=\Delta_{1}^{2}-\tau$. Hence in this case we have

$$
\xi_{1}+\xi_{2} \tau=\lambda_{5}{ }^{2}-\lambda_{6}{ }^{2} \tau, \quad\left(\lambda_{5} \text { and } \lambda_{6} \text { in } F\right) .
$$

Next let $\xi_{1}+1 \neq 0$, so that $\sigma \neq 0$; and let us define $\epsilon$ by the equation

$$
2 \sigma \epsilon=\xi_{2} .
$$

Then (24) gives $4 \sigma \pi=4 \sigma^{2} \epsilon^{2} \tau$, whence

$$
\pi=\epsilon^{2} \sigma \tau \text {. }
$$

But $2(\sigma-\pi)=\xi_{1}+1-\left(\xi_{1}-1\right)=2$, whence

$$
1=\sigma-\pi=\sigma-\epsilon^{2} \sigma \tau=\sigma\left(1-\epsilon^{2} \tau\right) .
$$

Since $1-\epsilon^{2} \tau \neq 0$, using Lemma 1 , we have 


$$
\sigma=\beta_{1}^{2}-\beta_{2}^{2} \tau
$$

so that $\xi_{1}=\pi+\sigma=\sigma\left(1+\epsilon^{2} \tau\right)$, and

$$
\begin{aligned}
\xi_{1}+\xi_{2} \tau & =\sigma\left[\left(1+\epsilon^{2} \tau\right)+2 \epsilon \tau\right]=\sigma\left[(1+\epsilon \tau)^{2}-(\epsilon \Delta)^{2} \tau\right] \\
& =\left(\beta_{1}{ }^{2}-\beta_{2}{ }^{2} \tau\right)\left[(1+\epsilon \tau)^{2}-(\epsilon \Delta)^{2} \tau\right]=\lambda_{5}{ }^{2}-\lambda_{6}{ }^{2} \tau
\end{aligned}
$$

for $\lambda_{5}$ and $\lambda_{6}$ in $F$, by Lemma 1 . Hence in all cases (25) is satisfied.

If we now put $\beta_{3}=\alpha_{1}+\tau \alpha_{2}, \beta_{4}=\Delta \alpha_{2}$, (22) becomes

$$
\delta\left(\lambda_{5}{ }^{2}-\lambda_{6}{ }^{2} \tau\right)=\beta_{3}{ }^{2}-\beta_{4}{ }^{2} \tau \text {. }
$$

Suppose first that $\beta_{3}{ }^{2}-\beta_{4}{ }^{2} \tau=0$, whence $\beta_{3}=\beta_{4}=0$. Then our definitions above of $\beta_{3}$ and $\beta_{4}$ evidently give $\alpha_{1}=\alpha_{2}=0$, and (20) take the form $\nu \tau=\delta \xi_{1},-\nu=\delta \xi_{2}$. Squaring each side of both these, we may write $\nu^{2} \tau^{2}=\delta^{2} \xi_{1}^{2}, \nu^{2} \tau=\delta^{2} \xi_{2}^{2} \tau$, whence, by subtraction and the use of the relations $1=\xi_{1}^{2}-\xi_{2}^{2} \tau, \tau=1+\Delta^{2}$, we obtain

$$
\nu^{2} \tau^{2}-\nu^{2} \tau=\tau\left(\nu^{2} \Delta^{2}\right)=\delta^{2}\left(\xi_{1}^{2}-\xi_{2}^{2} \tau\right)=\delta^{2} .
$$

Then $\tau=\left(\delta \nu^{-1} \Delta^{-1}\right)^{2}$, which is a contradiction since $\tau$ is not the square of any quantity of $F$. Hence $\beta_{3}{ }^{2}-\beta_{4}{ }^{2} \tau \neq 0$. Thus $\lambda_{5}{ }^{2}-\lambda_{6}{ }^{2} \tau \neq 0$ has an inverse in $F$ which has the form $\lambda_{f}{ }^{2}-\lambda_{8}{ }^{2} \tau$ by Lemma 1 , and we may write

$$
\begin{aligned}
\gamma & =\delta\left(\lambda_{3}{ }^{2}-\lambda_{4}{ }^{2} \tau\right)=\left(\lambda_{3}{ }^{2}-\lambda_{4}{ }^{2} \tau\right)\left(\lambda_{7}{ }^{2}-\lambda_{8}{ }^{2} \tau\right)\left(\beta_{3}{ }^{2}-\beta_{4}{ }^{2} \tau\right) \\
& =\left(\alpha^{2}-\beta^{2} \tau\right)
\end{aligned}
$$

again using Lemma 1 . We have proved in all cases the first part of the following statement.

Theorem 1. A scalar $\gamma \neq 0$ in $F$ has the property

$$
\gamma^{2}=N(a)
$$

for $a$ in $F(i)$, a cyclic quartic field, if and only if

$$
\gamma=\alpha^{2}-\beta^{2} \tau, \quad(\alpha \text { and } \beta \text { in } F),
$$

where $F(u)$ is the quadratic subfield of $F(i)$ defined by (1) and (3), and $u^{2}=\tau$.

Moreover, when $\gamma=\alpha^{2}-\beta^{2} \tau$, we have $N(\alpha+\beta \mu)=\left(\alpha^{2}-\beta^{2} \tau\right)^{2}$ $=\gamma^{2}$, which is the converse in the preceding theorem. 
Suppose now that $\gamma=\alpha^{2}-\beta^{2} \tau$ and $\gamma^{2}=N(b)$. If $a=\alpha+\beta u$, so that $\gamma=a a^{\prime}$, we have $\gamma^{2}=N(a)=N(b)$. It follows that $b \neq 0$ and $N\left(a b^{-1}\right)=1, a=w b$, where $N(w)=1$. Thus we have the following corollary.

Corollary 1. Let $\gamma=a a^{\prime}$, where $a$ is in $F(u)$. Then $\gamma^{2}=N(b)$ for $b$ in $F(i)$ if and only if $b$ is the product of $a$ by a unit of $F(i)$.

Since $-1=d d^{\prime}$, where $d$ is given in (16) and is in $F(u)$, we have also the following result.

COROLlary 2. The scalar $\gamma^{2}=N(b)$ for $b$ in $F(i)$ if and only if $-\gamma=e e^{\prime}$ for $e$ in $F(u)$.

3. The Wedderburn Norm Condition. For a cyclic algebra of order sixteen Wedderburn's condition becomes

$$
\gamma^{r} \neq N(a), \quad(r=1,2,3) .
$$

It is easily shown* that if $\gamma$ or $\gamma^{3}$ were a norm then $A$ would not be a division algebra. Hence the only possible case is $\gamma^{2}=N(a)$. By Theorem 1 this implies that $\gamma=\alpha^{2}-\beta^{2} \tau$. Consider the sub-algebra

$$
\sum=\left(y^{r}, u y^{r}\right), \quad(r=0,1,2,3),
$$

an algebra of order eight with $y u=-u y, y^{4}=\gamma, u^{2}=\tau$ in $F$, $y^{2} u=u y^{2}$. We shall write

$$
s=\left(e+y^{2}\right) y, t=i\left(a_{1}+y^{2}\right), \quad a_{1}=\beta_{1} q-\beta_{2} u,
$$

where we have used Corollary 2 to write

$$
-\gamma=e e^{\prime}, e=\beta_{1}+\beta_{2} u, \quad\left(\beta_{1} \text { and } \beta_{2} \text { in } F\right),
$$

and have

$$
y i=\theta(i) y, \theta(i)=q i, q=\Delta^{-1}(u+1), q q^{\prime}=-1,
$$

since $\Delta^{2} q q^{\prime}=(u+1)(-u+1)=-\left(1+\Delta^{2}\right)+1=-\Delta^{2}$. We shall compute

$$
\begin{aligned}
s t & =\left[\left(e+y^{2}\right) y\right]\left[i\left(a_{1}+y^{2}\right)\right]=\left(e+y^{2}\right) q i\left(a_{1}{ }^{\prime}+y^{2}\right) y \\
& =i q\left(e-y^{2}\right)\left(a_{1}{ }^{\prime}+y^{2}\right) y=i q\left[\left(e a^{\prime}-\gamma\right)+\left(e-a_{1}{ }^{\prime}\right) q y^{2}\right] y,
\end{aligned}
$$

since $y a=a^{\prime} y$ for every $a$ of $F(i)$ and $y^{2} i=-i y^{2}$. Now

* See the author's paper On direct products, etc., loc. cit. 


$$
\begin{aligned}
& q\left(e a^{\prime}-\gamma\right)=q\left(e a^{\prime}+e e^{\prime}\right)=e q\left(a_{1}^{\prime}+e^{\prime}\right) \\
& \quad=e q\left[\beta_{1} q^{\prime}+\beta_{2} u+\beta_{1}-\beta_{2} u\right]=\beta_{1} e\left[q q^{\prime}+q\right]=\beta_{1} e(q-1) .
\end{aligned}
$$

Also

$$
q\left(e-a_{1}^{\prime}\right)=q\left[\left(\beta_{1}+\beta_{2} u\right)-\left(\beta_{1} q^{\prime}+\beta_{2} u\right)\right]=\beta_{1}(q+1) .
$$

It follows that

$$
s t=\beta_{1} i\left[e(q-1)+(q+1) y^{2}\right] y .
$$

We have similarly

$$
\begin{gathered}
t s=\left[i\left(a_{1}+y^{2}\right)\right]\left[\left(e+y^{2}\right) y\right]=i\left[\left(a_{1} e+\gamma\right)+\left(a_{1}+e\right) y^{2}\right] y, \\
a_{1} e+\gamma=a_{1} e-e e^{\prime}=e\left[\left(\beta_{1} q-\beta_{2} u\right)-\left(\beta_{1}-\beta_{2} u\right)\right]=\beta_{1} e(q-1),
\end{gathered}
$$

while $a_{1}+e=\beta_{1} q-\beta_{2} u+\beta_{1}+\beta_{2} u=\beta_{1}(q+1)$. We then obtain immediately from (39)

$$
s t=t s \text {. }
$$

Consider the linear sets

$$
B=(1, u, s, u s), \quad C=\left(1, y^{2}, t, y^{2} t\right),
$$

over $F$. We have the relations

(42) $s u=-u s, t y^{2}=-y^{2} t ; u y^{2}=y^{2} u, u t=t u, s y^{2}=y^{2} s, s t=t s$, so that every quantity of $B$ is commutative with every quantity of $C$. We now show that

(43) $s^{2}=\left(e+y^{2}\right)\left(e^{\prime}+y^{2}\right) y^{2}=\left[\left(e e^{\prime}+\gamma\right)+\left(e+e^{\prime}\right) y^{2}\right] y^{2}=2 \beta_{1} \gamma$, since $e+e^{\prime}=2 \beta_{1}, e e^{\prime}=-\gamma$. Also

$$
\begin{aligned}
t^{2} & =i^{2}\left(a_{1}-y^{2}\right)\left(a_{1}+y^{2}\right)=i^{2}\left(a_{1}^{2}-\gamma\right) \\
& =i^{2}\left[\beta_{1}^{2} q^{2}-2 \beta_{1} \beta_{2} q u+{\beta_{2}}^{2} \tau+\beta_{1}^{2}-\beta_{2}^{2} \tau\right] \\
& =i^{2} \beta_{1}^{2}\left(q^{2}+1\right)-2 \beta_{1} \beta_{2} q u i^{2},
\end{aligned}
$$

since $\gamma=-e e^{\prime}$. We have also $i^{2}=\nu(u-\tau)$, so that

$$
\begin{aligned}
i^{2} q & =\nu \Delta^{-1}(u-\tau)(u+1)=\Delta^{-1} \nu(\tau-\tau+u-u \tau) \\
& =\Delta^{-1} \nu u(1-\tau)=\Delta^{-1} \nu u\left(-\Delta^{2}\right)=-\Delta \nu u .
\end{aligned}
$$

Moreover, we know that

$$
\begin{aligned}
i^{2}\left(q^{2}+1\right) & =i^{2}\left[\Delta^{-2}(\tau+2 u+1)+1\right]=\Delta^{-2} i^{2}\left[2 u+\tau+\left(1+\Delta^{2}\right)\right] \\
& =2 \nu \Delta^{-2}(u-\tau)(u+\tau)=2 \nu \Delta^{-2}\left(\tau-\tau^{2}\right)=-2 \nu \tau .
\end{aligned}
$$


Hence

$$
t^{2}=2 \nu \tau \beta_{1}\left(\beta_{2} \Delta-\beta_{1}\right) .
$$

We shall assume at this point that

$$
\beta_{1} \neq 0, \beta_{2} \Delta-\beta_{1} \neq 0,
$$

for otherwise either $s^{2}=0$ or $t^{2}=0$, and $A$ is evidently not a divi . sion algebra since from their form neither $s$ nor $t$ is zero. As a consequence, $a_{1}^{2}-\gamma \neq 0$ has an inverse in $F(u)$ and $e^{2}-\gamma \neq 0$ has an inverse in $F(u)$ since $t^{2}=i^{2}\left(a_{1}^{2}-\gamma\right) \neq 0$, while if

$$
e^{2}-\gamma=e^{2}-e e^{\prime}=0,
$$

then $e\left(e-e^{\prime}\right)=2 \beta_{1} e=0$, contrary to the hypothesis that $\beta_{1} \neq 0$, so that $e \neq 0$ has an inverse in $F(u)$. The sets $B$ and $C$ are generalized quaternion algebras over $F$, since in $B$

while in $C$

$$
u^{2}=\tau, s^{2}=2 \beta_{1} \gamma, s u=-u s,
$$

$$
\left(y^{2}\right)^{2}=\gamma, t^{2}=2 \nu \tau \beta_{1}\left(\beta_{2} \Delta-\beta_{1}\right), y^{2} t=-t y^{2},
$$

and evidently from the form of $s$ and $t$ the quantities 1, $u, s, u s$ are linearly independent in $F$, and the quantities $1, y^{2}, t, y^{2} t$ are linearly independent in $F$, when $i^{\alpha} y^{\beta}(\alpha, \beta=0,1,2,3)$ form a basis of $A$. The linear set $B C=C B$ of all sums of all products of quantities of $B$ and quantities of $C$ is an algebra, since a product

$$
\left(\sum_{\lambda} b_{1 \lambda} c_{1 \lambda}\right)\left(\sum_{\mu} b_{2 \mu} c_{2 \mu}\right)=\sum_{\lambda, \mu}\left(b_{1 \lambda} b_{2 \mu}\right)\left(c_{1 \lambda} c_{2 \mu}\right)
$$

is in $B C$ because for every $\lambda$ and $\mu$ the quantities $b_{1 \lambda} b_{2 \mu}$ are in $B$ and $C_{1 \lambda} C_{2 \mu}$ are in $C$. Now $B C$ contains $F(u)$ and hence $\left(\gamma-a_{1}^{2}\right)^{-1}$, $\left(\gamma-e^{2}\right)^{-1}$. Since $B C$ contains $s, t, e, a_{1}, y^{2}$, and is an algebra, it contains

$$
\begin{aligned}
\left(\gamma-a_{1}^{2}\right)^{-1}\left(t y^{2}-a_{1} t\right) & =\left(\gamma-a_{1}^{2}\right)^{-1} i\left(a_{1} y^{2}+\gamma-a_{1}^{2}-a_{1} y^{2}\right) \\
& =\left(\gamma-a_{1}^{2}\right)^{-1}\left(\gamma-a_{1}^{2}\right) i=i,
\end{aligned}
$$

and

$$
\begin{aligned}
\left(\gamma-e^{2}\right)^{-1}\left(y^{2} s-e s\right) & =\left(\gamma-e^{2}\right)^{-1}\left[\left(y^{2} e+\gamma\right)-e y^{2}-e^{2}\right] y \\
& =\left(\gamma-e^{2}\right)^{-1}\left(\gamma-e^{2}\right) y=y .
\end{aligned}
$$

But then $B C$ contains the basis of $A$ and has order sixteen. It follows that $A$ is the direct product of $B$ and $C$. 
THEOREM 2. Let $\gamma^{2}=N(a)$ for some a of $F(i)$ so that $-\gamma=e e^{\prime}$, where $e=\beta_{1}+\beta_{2} u$ and $\beta_{1}$ and $\beta_{2}$ are in $F$. Let $\beta_{1} \neq 0, \beta_{2} \Delta \neq \beta_{1}$, a set of necessary conditions that $A$ be a division algebra. Then the cyclic algebra $A$ is the direct product of two generalized quaternion algebras $B=(1, u, s, u s), C=(1, j, t, j t)$, with $y^{2}=j, s u=-u s$, $t j=-j t$, and

$$
u^{2}=\tau, s^{2}=2 \beta_{1} \gamma=\sigma, j^{2}=\gamma, t^{2}=\rho=2 \nu \beta_{1} \tau\left(\beta_{2} \Delta-\beta_{1}\right)
$$

Consider now the direct product of any two generalized quaternion algebras $B$ and $C$. It is known that $d$ in $B$ has the property that $d^{2}$ is in $F$ if and only if

$$
d=\lambda_{1} u+\lambda_{2} s+\lambda_{3} u s, d^{2}=Q_{1}=\lambda_{1}{ }^{2} \tau+\lambda_{2}{ }^{2} \sigma-\lambda_{3}{ }^{2} \sigma \tau,
$$

with $\lambda_{1}, \lambda_{2}$ and $\lambda_{3}$ in $F$. Similarly if $f$ is in $C$ then $f^{2}$ is in $F$ if and only if

$$
f=\lambda_{4} j+\lambda_{5} t+\lambda_{6} j t, f^{2}=Q_{2}=\lambda_{4}^{2} \gamma+\lambda_{5}^{2} \rho-\lambda_{6}^{2} \gamma \rho
$$

for $\lambda_{4}, \lambda_{5}$, and $\lambda_{6}$ in $F$. Suppose first that $Q \equiv Q_{1}-Q_{2}$ is a null form, that is, that we can make $Q=0$ for values of $\lambda_{1}, \cdots, \lambda_{6}$ in $F$ not all zero. Define $d$ by (47) and $f$ by (48) for the particular $\lambda_{i}$ we have used to make $Q$ vanish. Since $A$ is the direct product of $B$ and $C$, the quantities $d-f$ and $d+f$ are both not zero when the $\lambda_{i}$ are not all zero. But $(d-f)(d+f)=d^{2}-f^{2}=Q_{1}-Q_{2}=Q=0$. Hence in $A$ a product of two non-zero quantities is zero and $A$ is not a division algebra.

Conversely, let $Q$ not be a null form. Then, in particular, $Q_{1}$ and $Q_{2}$ are not null forms and $B$ and $C$ are known* to be division algebras. The algebra $\Gamma$ whose quantities have the form $X=x_{1}+x_{2} u$, where $x_{1}$ and $x_{2}$ are in $C$, has a division sub-algebra $C$ and the property that if we define $x^{\prime}=x$ for every $x$ of $C$, then $u^{2}=\tau$ in $C, x^{\prime \prime}=\left(x^{\prime}\right)^{\prime}=u^{2} x u^{-2}=x$ for every $x$ of $C$. But then $\Gamma$ is known $\dagger$ to be a division algebra if and only if $\tau \neq x^{\prime} x=x^{2}$ for any $x$ of $C$. But $\tau$ is in $F$ and if $\tau=x^{2}$ then, since $x$ is an $f$ of (48), and $u$ is a $d$ of (47), we have $Q=0$ for $\lambda_{1}=1$, a contradiction of our hypothesis that $Q$ was not a null form.

Define $X^{\prime}=x_{1}-x_{2} u$, for every $X$ of $\Gamma$, and we will have $X^{\prime}=s X s^{-1}, X^{\prime \prime}=s^{2} X s^{-2}=X, s^{2}=\sigma$ in $F$. Then it is known

* See L. E. Dickson, Algebren und ihre Zahlentheorie, p. 47, for the condition $\sigma \neq \xi_{1}{ }^{2}-\xi_{2}{ }^{2} \tau$, equivalent to the condition we have stated.

$\dagger$ A theorem of L. E. Dickson, ibid., pp. 63-64. 
(Dickson, loc. cit.) that $A$, whose quantities have the form $X+Y s$, is a division algebra when $\Gamma$ is one if and only if

$$
s^{2}=X^{\prime} X \text { for any } X \text { of } \Gamma \text {. }
$$

But if $s^{2}=X^{\prime} X,\left(x_{1}-x_{2} u\right)\left(x_{1}+x_{2} u\right)=x_{1}^{2}-x_{2}^{2} \tau+\left(x_{1} x_{2}-x_{2} x_{1}\right) u=\sigma$ we have

$$
\sigma=x_{1}^{2}-x_{2}^{2} \tau, x_{1} x_{2}=x_{2} x_{1} .
$$

First let $x_{1}$ and $x_{2}$ be in $F$. Then $Q$ is a null form when we take $\lambda_{1}=\sigma, \lambda_{2}=\tau x_{2}, \lambda_{3}=x_{1}, \lambda_{4}=\lambda_{5}=\lambda_{6}=0$, since $0=\sigma \tau\left(\sigma+x_{2}^{2} \tau-x_{1}^{2}\right)$ $=\sigma^{2} \tau+\left(\tau x_{2}\right)^{2} \sigma-x_{1}^{2} \sigma \tau$, a contradiction. Next let $x_{1}$ be in $F$ but $x_{2}$ not in $F$. Then $x_{2}{ }^{2} \tau=x_{1}{ }^{2}-\sigma$ is in $F$ and $x_{2} \tau$ is an $f$ of (48) while $\left(x_{2} \tau\right)^{2}=Q_{2}=x_{1}^{2} \tau-\sigma \tau$ so that $Q$ is a null form for $Q_{2}=\left(x_{2} \tau\right)^{2}$, $\lambda_{1}=x_{1}, \lambda_{2}=0, \lambda_{3}=1$. The only remaining case is where $x_{1}$ is not in $F$. If $x_{1}^{2}$ were in $F$ so that $x_{1}$ would be an $f$ of (48), then $x_{1} x_{2}$ $=x_{2} x_{1}$ implies that $x_{2}=\xi+\eta x_{1}, \xi$ and $\eta$ in $F$, since in a generalized quaternion division algebra the only quantities commutative with a non-scalar quantity $x$ are scalar coefficient polynomials in $x$. But $x_{2}^{2}$ is in $F$ so that $\eta=0$ or $\xi=0$. When $\eta=0$, then $x_{1}{ }^{2}=\xi^{2} \tau+\sigma$ and $Q_{2}=Q_{1}=\xi^{2} \tau+1^{2} \sigma-O \sigma \tau$, a contradiction of our hypothesis. When $\xi=0$ then $x_{2}=\eta x_{1}$ and $x_{1}^{2}-x_{2}^{2} \tau$ $=x_{1}{ }^{2}\left(1-\eta^{2} \tau\right)$. But by Lemma 1 we have $\left(1-\eta^{2} \tau\right)^{-1}=\delta_{1}{ }^{2}-\delta_{2}{ }^{2} \tau$ and $x_{1}^{2}=Q_{2}=\sigma \delta_{1}^{2}-\sigma \tau \delta_{2}^{2}=Q_{1}$, a contradiction. We have finally come to the case where neither $x_{1}$ nor its square is in $F$. We then have, where $f$ is given by (47) and $f^{2}=Q_{2}$, that $x_{1}=\lambda_{7}+f$ with $\lambda_{7} \neq 0$ in $F$. As before the relation $x_{1} x_{2}=x_{2} x_{1}$ implies that $x_{2}$ is a polynomial in $x_{1}$. But now we may write $x_{2}=\xi+\eta f$. Now

$$
x_{1}^{2}-x_{2}{ }^{2} \tau=\lambda_{7}{ }^{2}+2 \lambda_{7} f+Q_{2}-\left(\xi^{2}+2 \xi \eta f+\eta^{2} Q_{2}\right) \tau=\sigma .
$$

It follows that $2 \lambda_{7}-2 \xi \eta \tau=0$, so that $\lambda_{7}=\xi \eta \tau$ and

$$
\sigma=\xi^{2} \eta^{2} \tau^{2}-\xi^{2} \tau+Q_{2}\left(1-\eta^{2} \tau\right)=\left(Q_{2}-\xi^{2} \tau\right)\left(1-\eta^{2} \tau\right) .
$$

The quantity $\left(1-\eta^{2} \tau\right) \neq 0$ has an inverse $\delta_{1}^{2}-\delta_{2}^{2} \tau$ with $\delta_{1}$ and $\delta_{2}$ in $F$ by Lemma 1 , and $Q_{2}-\xi^{2} \tau=\sigma\left(\delta_{1}{ }^{2}-\delta_{2}{ }^{2} \tau\right)$, so that we have $Q_{2}=\xi^{2} \tau+\sigma \delta_{1}{ }^{2}-\sigma \tau \delta_{2}{ }^{2}=Q_{1}$. We have again shown that if $A$ were not a division algebra, then $Q$ would be a null form, a contradiction of our hypothesis. Hence $A$ is a division algebra and we have proved the following theorem 
Theorem 3. $A$ direct product $A=B \times C$ of two generalized quaternion algebras $B=(1, u, s, u s), C=(1, j, t, j t)$ with $u^{2}=\tau$, $s^{2}=\sigma, s u=-u s, j^{2}=\gamma, t^{2}=\rho, t j=-j t$, is a division algebra if and only if the quadratic form

$$
Q=\left(\lambda_{1}{ }^{2} \tau+\lambda_{2}{ }^{2} \sigma-\lambda_{3}{ }^{2} \sigma \tau\right)-\left(\lambda_{4}{ }^{2} \gamma+\lambda_{5}{ }^{2} \rho-\lambda_{6}{ }^{2} \gamma \rho\right)
$$

in the variables $\lambda_{1}, \lambda_{2}, \cdots, \lambda_{6}$ in $F$, is not a null form.

We may now apply Theorem 3 and our previous results to obtain complete necessary and sufficient conditions that a cyclic algebra be a division algebra. We first assume that $\gamma^{2}$ $=N(a)$ for some $a$ in $F(i)$. If $\gamma=0$, then $-\gamma=\beta_{1}{ }^{2}-\beta_{2}{ }^{2} \tau$ with $\beta_{1}=\beta_{2}=0$, and the form $Q$ may be defined. If $\gamma \neq 0$, then by Corollary 2 we can again define the form $Q$ with

$$
\sigma=2 \beta_{1} \gamma, \rho=2 \nu \tau \beta_{1}\left(\beta_{2} \Delta-\beta_{1}\right) \text {. }
$$

Suppose first that $Q$ is a null form. If $\gamma=0$, then $y^{4}=0$ while $y$ is not zero and $A$ is not a division algebra. If $\gamma \neq 0$ but $\beta_{1}=0$ or $\beta_{2} \Delta-\beta_{1}=0$, then again, as we have seen, $A$ is not a division algebra. The only other case is where Theorem 2 can be applied and, by Theorem $3, A$ is again not a division algebra. Conversely let $Q$ be not a null form. Then obviously from our definition of $Q$ as above and the fact that we have the coefficients of $Q$ all not zero in a non-null form, $\gamma \neq 0, \beta_{2} \Delta \neq \beta_{1}, \beta_{1} \neq 0$, and again $A$ is the direct product of $B$ and $C$; we may again apply Theorem 3 , and $A$ is a division algebra.

THEOREM 4 . Let $A$ be a cyclic algebra with basis $i^{\lambda} y^{\mu},(\lambda, \mu=0$, $1,2,3)$, where $i$ is a root of the cyclic quartic

$$
\phi(\omega) \equiv \omega^{4}+2 \nu \tau \omega^{2}+\nu^{2} \Delta^{2} \tau=0
$$

with $\tau=1+\Delta^{2}, \nu \neq 0, \Delta \neq 0$ in $F$, and $\tau$ not the square of any element in $F$. Also

$$
\theta(i)=q i, q \Delta=1+u, i^{2}=\nu(u-\tau), y i=\theta(i) y, y^{4}=\gamma i n F .
$$

Suppose that $\gamma^{2}$ is the norm of a quantity of $F(i)$ so that we have $-\gamma=\beta_{1}^{2}-\beta_{2}^{2} \tau$ with $\beta_{1}$ and $\beta_{2}$ in $F$. Then $A$ is a division algebra if and only if the form

$$
Q=\lambda_{1}{ }^{2} \tau+\lambda_{2}{ }^{2} \sigma-\lambda_{3}{ }^{2} \sigma \tau-\lambda_{4}{ }^{2} \gamma-\lambda_{5}{ }^{2} \rho+\lambda_{6}{ }^{2} \gamma \rho
$$

with $\sigma=2 \beta_{1} \gamma, \rho=2 \beta_{1} \nu \tau\left(\beta_{2} \Delta-\beta_{1}\right)$ does not vanish for any $\lambda_{1}, \cdots$, $\lambda_{6}$ not all zero and in $F$. 
The only other case is $\gamma^{2} \neq N(a)$. Then obviously $\gamma \neq N(a)$ since otherwise $\gamma^{2}=N\left(a^{2}\right)$, a contradiction. If $\gamma^{8}=N(a)$, then either $\gamma=0$, whence $\gamma^{2}=N(0)$, a contradiction, or else $\gamma \neq 0$, $\gamma^{6}=\gamma^{2} \gamma^{4}=N\left(a^{2}\right), \gamma^{2}=N\left(a \gamma^{-1}\right)$, again a contradiction. Hence the condition $\gamma^{2} \neq N(a)$ is equivalent to the Wedderburn norm condition. We have also shown the former condition equivalent to the condition $\gamma \neq-e e^{\prime}$ for any $e$ of $F(u)$. We thus have proved the following theorem

TheOREM 5. Let all the hypotheses of Theorem 4 be satisfied except that now $\gamma^{2} \neq N(a)$ for any a of $F(i)$, or, what is the same thing, $-\gamma$ is not expressible in the form $\beta_{1}{ }^{2}-\beta_{2}{ }^{2} \tau, \beta_{1}$ and $\beta_{2}$ in $F$. Then the cyclic algebra $A$ is a division algebra.

We shall finally pass to the case where $F$ is the field $R$ of all rational numbers. Quadratic forms have been studied in detail for this case and it has been shown that every indefinite quadratic form in five or more variables is a null form.* The numbers $\tau, \sigma,-\sigma \tau$ all have the same sign only when all are negative. If they are all negative and $\gamma, \rho,-\gamma \rho$ are also all negative then $\tau$ and $-\gamma$ have opposite signs so that $Q=Q_{1}-Q_{2}$ is an indefinite quadratic form. In the other cases obviously $Q$ is indefinite, providing that its coefficients are all not zero. When some of the coefficients of $Q$ are zero then, by making all the other variables zero and those with zero coefficients not zero, we can make $Q$ zero so that $Q$ is a null form. When none of the coefficients of $Q$ is zero then $Q$ is an indefinite quadratic form in six variables and hence is a null form. Hence in every case the cyclic algebra $A$ is not a division algebra when the hypotheses of Theorem 4 are satisfied. We have the following result.

THEOREM 6. When $F=R$, the field of all rational numbers, the Wedderburn norm condition for cyclic algebras of order sixteen is necessary as well as sufficient.

\section{Columbia University}

* For the first complete proof of this theorem see L. E. Dickson, Studies in the Theory of Numbers.

$\dagger$ We also have here a new short proof of the author's theorem that a direct product of two rational generalized quaternion division algebras is never a division algebra, by using the above proof that when $Q$ is a null form $A$ is not a division algebra. This theorem was first proved by the author and published in the Annals of Mathematics, vol. 30 (1929), pp. 621-625. 